\title{
A Preliminary Study on the Mechanism of Premature Ovarian Failure
}

\author{
Tian Tang1*, Jinghan Yang2, Siyuan Jing² \\ ${ }^{1}$ Center for Reproductive Medicine, Department of Gynecology and Obstetrics, West China Second University Hospital, \\ Key Laboratory of Birth Defects and Related Diseases of Women and Children, Ministry of Education, Sichuan University, \\ Chengdu 610041, Sichuan Province, China
}

${ }^{2}$ West China School of Medicine, Sichuan University, Chengdu 610041, Sichuan Province, China

*Corresponding author: Tian Tang, tangsisley@sina.com

\begin{abstract}
Premature ovarian failure (POF) refers to the failure of ovaries which causes amenorrhea before the age of 40. It is characterized by primary or secondary amenorrhea along with elevated blood gonadotropin levels and decreased estrogen levels with varying degrees of low estrogen symptoms such as hot flashes and sweating, facial flushing, reduced libido, and so on. Premature ovarian failure (POF) is a common endocrine disease in gynecology. The incidence of POF is increasing year by year and the affected population increasingly involves the younger-aged groups. Its etiology is complex and diverse involving many factors such as genetics, immunity, medical treatment, environment endocrine disruptors, etc. It is also affected by long-term smoking, alcohol intake, and other behaviors which damage patients' physical and mental health. In recent years, the effects of gene therapy in restoring ovarian function and improving fertility in animal experiments have provided a new direction for POF patients. This article discusses the etiology and possible pathogenesis of POF and BRCA from various aspects as well as summarizes the recent research status and progress of new treatment methods of POF.
\end{abstract}

Keywords: Premature ovarian failure (POF); BRCA

Publication date: July 2021; Online publication: July 30, 2021

\section{Introduction}

Premature ovarian failure (POF) refers to women who are below 40 years old that have ovarian failure due to depletion of follicles in the ovaries or iatrogenic injury. Premature ovarian failure is multi-causal characterized by low estrogen and high gonadotropin levels. Amenorrhea or oligomenorrhea is accompanied by a series of perimenopausal symptoms. The etiology of premature ovarian failure includes genetic factors, autoimmune factors, enzymatic disorders, low primordial ovarian reserve, atresia or rapid depletion of follicles, iatrogenic factors, environmental or infectious factors, and psychological factors. It is often with different degrees of perimenopausal symptoms such as sparse or irregular menstruation, hot flashes and sweating, facial flushing, low libido, etc. Decreased ovarian function and premature ovarian failure affect the health and quality of life in majority of the affected women. In recent years, the incidence rate has been increasing but the treatment effects have not been satisfactory. This article reviews the etiology research related to BRCA gene.

\section{Research significance of premature ovarian failure}

According to reports at home and abroad, the decrease in the number and/or quality of oocytes is an important factor that causes low fertility and even infertility in women ${ }^{[1-2]}$. At present, in reality, judging from a large number of clinical situations, more and more women in the childbearing age have been 
diagnosed with POF resulting in reduced reproductive potential and the early loss of normal fertility ${ }^{[3]}$. At the same time, the age of onset of POF patients with fertility needs is also decreasing showing a youngerage trend and the issue of more effective treatments has become much more difficult ${ }^{[2-3]}$.

For a long time, treatment methods such as assisted reproductive technology (ART), in vitro fertilization-embryo transfer (IVF-ET), and fertility preservation have been used worldwide to assist such patients to conceive ${ }^{[4]}$. However, a large number of multicenter studies have pointed out that when POF patients receive ART treatment, the ovarian response is poor, the cycle cancellation rate is high, the use of ovulation induction drugs increases, the number of eggs obtained is minimal, the clinical pregnancy rate and the live-birth rate are low, and the abortion rate is high ${ }^{[5-6]}$. In addition, the cases conceived by using in vitro fertilization-embryo transfer have major ethical risks and other disadvantages. The above treatment options greatly increase the economic and psychological burden among patients ${ }^{[5-6]}$. At present, the latest treatment methods such as in vitro follicle activation, immunity, stem cells, gene editing, and other technologies are still in the preclinical stage where there is no definite clinical treatment effect. The issue on how to effectively improve the reproductive outcome among POF patients is a huge practical problem in the field of assisted reproduction. Therefore, from a medical point of view, reviewing the pathogenesis and treatment of POF, effectively intervening in ovarian reserve failure and reducing egg loss, thereby delaying ovarian aging and prolonging their reproductive life play vital roles in the health effect of women.

\section{Research status of BRCA gene and POF}

On the one hand, the BRCA gene family includes BRCA1 and BRCA2. They belong to the ataxiatelangiectasia mutated mediated (ATM-mediated) DNA double-strand break (DSB) damage repair genes. These genes may play key roles in the protection of DNA integrity in oocytes ${ }^{[7]}$. BRCA1 is an important gene for repairing double-stranded DNA damage. Its mutation may cause genetic instability of oocytes which in turn leads to impaired ovarian reserve that denotes the quantity and quality of remaining oocytes. On the other hand, women's potential for pregnancy depends on their ovarian reserve. Anti-Müllerian hormone $(\mathrm{AMH})$ is secreted by preantral follicles and granulosa cells around the early antral follicles. Its physiological function is mainly to inhibit the development of the early-stage follicles ${ }^{[8]}$. At present, AMH is considered to be the best biomarker which reflects the ovarian reserve function ${ }^{[8]}$. A new clinical study used linear regression to compare the differences in serum AMH levels between BRCA1 mutation carriers, BRCA2 mutation carriers, and non-carriers ${ }^{[9]}$. The results showed that BRCA1 mutation carriers have higher AMH levels than those in the non-carrier group which is $25 \%$ lower $(95 \% \mathrm{CI}=0.59-0.95, \mathrm{P}<0.05)$. However, there is no significant difference between the AMH levels in the BRCA2 mutation carrier group with the non-carrier group $(95 \% \mathrm{CI}=0.77-1.27, \mathrm{P}=-0.94)$. With the continuous improvement of detection technology, the detection rate of female BRCA1 mutation carriers in the reproductive period has been increasing year by year in China and the number of patients with BRCA1 mutations has been increasing. Studies have shown that mutations in the BRCA1 gene may cause POF. However, the correlation between BRCA1 mutation, accumulation of oocyte damage, POF, and IVF assisted pregnancy rate is not yet clear, hence further research is urgently needed.

\section{Correlation between BRCA1 mutation, double-strand breaks (DSBs) in the DNA of oocytes, and reproductive aging}

From embryonic primary oocytes to puberty, the first meiosis is completed in batches and stages. During this long quiescent period, it is important to ensure the integrity of the oocytes' genes or chromosomes. Once the genes or chromatins are damaged, the oocytes may become senescent or even undergo apoptosis. The aging of oocytes has become one of the important factors of female infertility. The common causes of 
oocyte senescence include genetic factors, iatrogenic factors, immune factors, environmental factors, etc. ${ }^{[10]}$. Covalent cross-links between DNA strands of oocytes can easily block DNA replication, transcription, and recombination leading to DNA double-strand breaks. The lack of crosslink repair ability between DNA strands in oocytes is also one of the reasons for abnormal changes in cells ${ }^{[11-12]}$. Among them, the most severe DNA damage in oocytes is DNA double-strand breaks which can easily kill replicating cells ${ }^{[11,13]}$. As early as 2001, Garcia et al. found that patients with Fanconi anemia have abnormal DSBs repair function and often presented with early menopause or premature hypogonadism ${ }^{[14]}$. Studies have shown that the most important mutant pathogenic gene, FANCD1 in Fanconi anemia is actually the BRCA1 gene. Another active form of gene, FANCD2 in its pathogenesis is also closely related to BRCA1. BRCA1 and its related pathogenic mutations may be an important cause of the abnormality in the repair mechanism of patients' DSBs $^{[15-16]}$. From this, it can be inferred that BRCA1 is an important gene for repairing double-stranded DNA damage and its mutation causes the instability of the genetic material in cells which leads to oocyte senescence or even apoptosis.

\section{Potential regulatory mechanism of BRCA1 gene in repairing DNA double-strand breaks (DSBs) in oocytes}

The subsequent research by Kutluk Oktay's team further verified the above hypothesis and pointed out that the DSBs repair protein related to BRCA1 is important for the maintenance of the lifespan of germ cells and the protection of ovarian function ${ }^{[17-19]}$. The team found that: 1 . With age, the expression of DNA repair-related genes in the ovaries of mice decreases; 2 . With aging, the expression of the oocyte DNA double-strand break damage marker, $\gamma \mathrm{H} 2 \mathrm{AX}$ significantly increases in mice; 3 . With aging, DNA doublestrand breaks significantly increase in the nucleus of oocytes and DNA double-strand breaks damage that have not been properly repaired as well as germ cell DNA damage continue to accumulate. The study proposed: 1. With age, quiescent oocytes continue to suffer DNA double-strand breaks; 2. BRCAl and related DSBs repair protein expression is negatively correlated with age; 3 . The failure of DNA damage repair causes loss of oocytes reserve. In order to confirm this speculation, they did a BRCA1 knockout in mice and found that the BRCA1 knockout mice showed significantly lower ovarian function than that of the wild-type mice in addition to the occurrence of premature ovarian failure. At the same time, Chen Zijiang et al. used whole exome sequencing technology to find a new BRCA2 complex in a sister with premature ovarian insufficiency (POI) and a sporadic POI patient. Studies have suggested that insufficient BRCA2 caused by pathogenic mutations can lead to isolated ovarian dysfunction ${ }^{[20]}$.

Carrying BRCA1 not only increases the risk of cancer but also poses a great threat to the repair of DNA damage in oocytes and fertility ${ }^{[1]}$. BRCA1 protein has domains related to DNA damage repair whereby abnormalities in any of the domains may cause DNA repair dysfunction which would increase the cumulative risk of oocyte damage and affect genetic stability ${ }^{[21]}$. These findings provide further evidence to the important role of BRCA genes in DNA damage repair of oocytes and ovarian function maintenance.

\section{Hypothesis of synergistic regulation mechanism in the upstream MRN protein complex of BRCA1 and the downstream RAD52 protein complex}

The specific mechanism of BRCA1 gene in oocytes against premature ovarian failure is still unclear which is mainly reflected in the following two aspects:

One is that the upstream signaling pathway that activates BRCA1 in oocytes is still unclear. According to previous studies, the three proteins which are MRE11, RAD50, and NBS1 constitute the core spherical domain of the MRN complex which may have effect in maintaining the stability of cells' genetic material. According to related studies by Kutluk Oktay et al., the nuclease activity of the MRN complex 
(MRE11/RAD50/NBS1 complex) and the nuclease of MRE11 alone may activate BRCA1 to bind to DNA [17-18]. These clinical phenomena and cellular characteristics suggest the importance of MRN complex in the activation of BRCA1 protein in oocytes and the signal transmission of DNA double-strand break damage repair. Based on the pre-experimental results, it has been speculated that the upstream pathway may activate the ATM pathway after cellular DNA double-strand breaks while the MRN complex acts as an ATM effector and downstream activates BRCA1.

Second, the downstream effect pathways of BRCA1 are not yet clear. There may be key proteins such as BRCA2 and RAD51 downstream of BRCA1 which bind, alloster, and affect the assembly of DNA damage repair elements. However, the mechanism of how BRCA1 interacts with downstream BRCA2, RAD51, and other proteins in the ovarian microenvironment as well as how the downstream completes the repair of oocytes' DNA double-strand breaks have not been fully understood. The preliminary experiment results found that POF patients have mutations such as MRE11/RAD50/NBS1/BRCA1/BRCA2/RAD51 and there are high-frequency mutations in the BRCA1 gene (e.g., rs799917, rs16941, rs16942). It is speculated that the BRCA1 gene mutation is missing. Aging is the main reason for the decline of ovarian function reserve and the mutations of key factors upstream and downstream together aggravate the DSBs repair. This inference and the conclusions of the literature are mutually corroborated. According to a few literatures, it has been suggested that the activation of BRCA1 and its downstream BRCA2 and RAD51 proteins may play important roles in the repair of oocytes' DNA double-strand break damage and recombination. It is speculated that the activated BRCA1 may be effective in the following two aspects. First, by working with MRN complex to complete the cutting of damaged DNA and second, by further activating BRCA2 and recruiting RAD51 to form a repair complex at the DNA damage site in cooperation with DNA polymerase to complete the DNA repair.

Therefore, the following hypotheses have been suggested:

1. DNA double-strand breaks trigger the MRN complex (MRE11/RAD50/NBS1 complex) in the oocytes of humans and rodents which activates BRCA1 and plays a key role in the repair of homologous recombination upstream effect.

2. The BRCA1 protein activates BRCA2 and RAD51 protein (RAD52 protein complex) which play a key role in the homologous recombination step of the DNA repair process and is important for maintaining the oocyte reserve in the ovaries of humans and mice.

Based on previous studies, the BRCA1 gene belongs to the ATM-mediated DNA DSBs repair gene family. There is a close relationship between BRCA1 mutation, DNA double-strand damage of oocytes, and reproductive senescence. It is believed that after DNA damage is recognized and amplified by ATM, BRCA1 may be activated under the cooperation of MRE11/RAD50/NBS1. In addition, BRCA1 activates BRCA2 and RAD51 proteins, assembles RAD52 protein repair elements, repairs oocyte DSBs in the form of homologous recombination, and maintains normal ovarian reserve function.

If the above-mentioned scientific hypotheses are confirmed, it is further expected to reveal the pathogenesis of decreased ovarian reserve, explore the repairing effect of BRCA1-related oocyte DSBs, and provide a new method for the comprehensive treatment of POF. It is hoped that by revealing the key influence of BRCA1 gene on ovarian function, scientifically predicting female reproductive aging would play an important role in prolonging the reproductive life of women.

\section{Funding}

This study is supported by the National Natural Science Foundation of China for Young Scholars (82003865), Application research of the Science and Technology Department in Sichuan Province (21YYJC2810 (2021YJ0461)), and Science and Technology Project of Chengdu: Key Research and Development Support Plan (2019-YF05-00250-SN). 


\section{Disclosure statement}

The authors declare that there is no conflict of interest.

\section{Author contributions}

Tian Tang contributed to the acquisition of funding, methodology, supervision, formal analysis, review, and editing. Jinghan Yang and Siyuan Jing contributed to the visualization, writing of the original draft, formal analysis, methodology, and supervision.

\section{References}

[1] De Pedro M, Otero B, Martin B, 2015, Fertility Preservation and Breast Cancer: A Review. Ecancermedicalscience, 9: 503.

[2] Rasool S, Shah D, 2017, Fertility with Early Reduction of Ovarian Reserve: The Last Straw that Breaks the Camel's Back. Fertil Res Pract, 3: 15.

[3] Tugrul Ayanoglu B, Ozdemir ED, et al., 2018, Diminished Ovarian Reserve in Patients with Psoriasis. Taiwan J Obstet Gynecol, 57(2): 227-30.

[4] Shandley LM, Fothergill A, Spencer JB, et al., 2018, Impact of Cancer Treatment on Risk of Infertility and Diminished Ovarian Reserve in Women with Polycystic Ovary Syndrome. Fertil Steril, 109(3): 516-25.

[5] Morin SJ, Patounakis G, Juneau CR, et al., 2018, Diminished Ovarian Reserve and Poor Response to Stimulation in Patients $<38$ Years Old: A Quantitative but Not Qualitative Reduction in Performance. Hum Reprod.

[6] Youssef MA, van Wely M, Mochtar M, et al., 2018, Low Dosing of Gonadotropins in In Vitro Fertilization Cycles for Women with Poor Ovarian Reserve: Systematic Review and Meta-Analysis. Fertil Steril, 109(2): 289-301.

[7] Knittel G, Rehkamper T, Nieper P, et al., 2018, DNA Damage Pathways and B-cell lymphomagenesis. Curr Opin Hematol, 25(4): 315-22.

[8] de Kat AC, Broekmans FJ, Laven JS, et al., 2015, Anti-Mullerian Hormone as a Marker of Ovarian Reserve in Relation to Cardio-Metabolic Health: A Narrative Review. Maturitas, 80(3): 251-7.

[9] Wang ET, Pisarska MD, Bresee C, et al., 2014, BRCA1 Germline Mutations may be Associated with Reduced Ovarian Reserve. Fertil Steril, 102(6): 1723-8.

[10] Babayev E, Wang T, Szigeti-Buck K, et al., 2016, Reproductive Aging is Associated with Changes in Oocyte Mitochondrial Dynamics, Function, and MtDNA Quantity. Maturitas, 93: 121-30.

[11] Wang ZW, Ma XS, Ma JY, et al., 2013, Laser Microbeam-Induced DNA Damage Inhibits Cell Division in Fertilized Eggs and Early Embryos. Cell Cycle, 12(20): 3336-44.

[12] Jia ZZ, Zhang JW, Zhou D, et al., 2019, Deltamethrin Exposure Induces Oxidative Stress and Affects Meiotic Maturation in Mouse Oocyte. Chemosphere, 223: 704-13.

[13] Kusakabe H, Tateno H, 2011, Characterization of Chromosomal Damage Accumulated in FreezeDried Mouse Spermatozoa Preserved Under Ambient and Heat Stress Conditions. Mutagenesis, 26(3): 447-53.

[14] Garcia-Higuera I, Taniguchi T, Ganesan S, et al., 2001, Interaction of the Fanconi Anemia Proteins and BRCA1 in a Common Pathway. Mol Cell, 7(2): 249-62.

[15] Brosh RM, Jr., Bellani M, Liu Y, et al., 2017, Fanconi Anemia: A DNA Repair Disorder Characterized 
by Accelerated Decline of the Hematopoietic Stem Cell Compartment and Other Features of Aging. Ageing Res Rev, 33: 67-75.

[16] Garcia-Muse T, Boulton SJ, 2005, Distinct Modes of ATR Activation After Replication Stress and DNA Double-Strand Breaks in Caenorhabditis Elegans. EMBO J, 24(24): 4345-55.

[17] Oktay K, Kim JY, Barad D, et al., 2010, Association of BRCA1 Mutations with Occult Primary Ovarian Insufficiency: A Possible Explanation for the Link Between Infertility and Breast/Ovarian Cancer Risks. J Clin Oncol, 28(2): 240-4.

[18] Lin W, Titus S, Moy F, et al., 2017, Ovarian Aging in Women with BRCA Germline Mutations. J Clin Endocrinol Metab, 102(10): 3839-47.

[19] Oktay K, Harvey BE, Loren AW, 2018, Fertility Preservation in Patients with Cancer: ASCO Clinical Practice Guideline Update Summary. J Oncol Pract, 14(6):381-5.

[20] Qin FZ, Chen Z, 2019, BRCA2 in Ovarian Development and Function. New Engl J Med, (380): 10867.

[21] Lee JH, Mand MR, Kao CH, et al., 2018, ATM directs DNA Damage Responses and Proteostasis Via Genetically Separable Pathways. Sci Signal, 11(512).

[22] Shen SX, Weaver Z, Xu X, et al., 1998, A Targeted Disruption of the Murine BRCA1 Gene Causes Gamma-Irradiation Hypersensitivity and Genetic Instability. Oncogene, 17(24): 3115-24.

[23] Huber LJ, Yang TW, Sarkisian CJ, et al., 2001, Impaired DNA Damage Response in Cells Expressing an Exon 11-Deleted Murine BRCA1 Variant that Localizes to Nuclear Foci. Mol Cell Biol, 21(12): 4005-15. 